\title{
A viscosity approximation method for weakly relatively nonexpansive mappings by the sunny nonexpansive retractions in Banach spaces
}

\author{
Chin-Tzong Pang ${ }^{1}$, Eskandar Naraghirad ${ }^{2 *}$ and Ching-Feng Wen ${ }^{3}$
}

\author{
"Correspondence: \\ eskandarrad@gmail.com; \\ esnaraghirad@mail.yu.ac.ir \\ 2 Department of Mathematics, \\ Yasouj University, Yasouj, 75918, Iran \\ Full list of author information is \\ available at the end of the article
}

\begin{abstract}
In this paper, we introduce a new viscosity approximation method by using the shrinking projection algorithm to approximate a common fixed point of a countable family of nonlinear mappings in a Banach space. Under quite mild assumptions, we establish the strong convergence of the sequence generated by the proposed algorithm and provide an affirmative answer to an open problem posed by Maingé (Comput. Math. Appl. 59:74-79, 2010) for quasi-nonexpansive mappings. In contrast with related processes, our method does not require any demiclosedness principle condition imposed on the involved operators belonging to the wide class of quasi-nonexpansive operators. As an application, we also introduce an iterative algorithm for finding a common element of the set of common fixed points of an infinite family of quasi-nonexpansive mappings and the set of solutions of a mixed equilibrium problem in a real Banach space. We prove a strong convergence theorem by using the proposed algorithm under some suitable conditions. Our results improve and generalize many known results in the current literature.

MSC: $47 \mathrm{H} 10 ; 37 \mathrm{C} 25$
\end{abstract}

Keywords: viscosity approximation method; fixed point; weak relatively nonexpansive mapping; strong convergence

\section{Introduction}

Throughout this paper, the set of real numbers and the set of positive integers are denoted by $\mathbb{R}$ and $\mathbb{N}$, respectively. Let $E$ be a Banach space with the norm $\|\cdot\|$ and the dual space $E^{*}$. When $\left\{x_{n}\right\}_{n \in \mathbb{N}}$ is a sequence in the Banach space $E$, we denote the strong convergence of $\left\{x_{n}\right\}_{n \in \mathbb{N}}$ to $x \in E$ by $x_{n} \rightarrow x$ and the weak convergence by $x_{n} \rightarrow x$. For any sequence $\left\{x_{n}^{*}\right\}_{n \in \mathbb{N}}$ in $E^{*}$, we denote the strong convergence of $\left\{x_{n}^{*}\right\}_{n \in \mathbb{N}}$ to $x^{*} \in E^{*}$ by $x_{n}^{*} \rightarrow x^{*}$, the weak convergence by $x_{n}^{*} \rightarrow x^{*}$ and the weak-star convergence by $x_{n}^{*} \rightarrow^{*} x^{*}$. The modulus of convexity of a Banach space $E$ is defined by

$$
\delta(\epsilon)=\inf \left\{1-\frac{\|x+y\|}{2}:\|x\| \leq 1,\|y\| \leq 1,\|x-y\| \geq \epsilon\right\}
$$

for every $\epsilon$ with $0 \leq \epsilon \leq 2$. A Banach space $E$ is said to be uniformly convex if $\delta(\epsilon)>0$ for every $\epsilon>0$. Let $S_{E}=\{x \in E:\|x\|=1\}$. The norm of $E$ is said to be Gâteaux differentiable if

\section{Springer}

(c) 2015 Pang et al.; licensee Springer. This is an Open Access article distributed under the terms of the Creative Commons Attribution License (http://creativecommons.org/licenses/by/4.0), which permits unrestricted use, distribution, and reproduction in any medium, provided the original work is properly credited. 
for each $x, y \in S_{E}$, the limit

$$
\lim _{t \rightarrow 0} \frac{\|x+t y\|-\|x\|}{t}
$$

exists. In this case, $E$ is called smooth. If the limit (1.1) is attained uniformly in $x, y \in S_{E}$, then $E$ is called uniformly smooth. The Banach space $E$ is said to be strictly convex if $\left\|\frac{x+y}{2}\right\|<1$ whenever $x, y \in S_{E}$ and $x \neq y$. It is well known that $E$ is uniformly convex if and only if $E^{*}$ is uniformly smooth. It is also known that if $E$ is reflexive, then $E$ is strictly convex if and only if $E^{*}$ is smooth; for more details, see [1]. The normalized duality mapping $J: E \rightarrow 2^{E^{*}}$ is defined by

$$
J(x)=\left\{f \in E^{*}:\langle x, f\rangle=\|x\|^{2},\|x\|=\|f\|\right\}, \quad \forall x \in E .
$$

If a Banach space $E$ admits a sequentially continuous duality mapping $J$ from weak topology to weak-star topology, then $J$ is single-valued and also $E$ is smooth; see for more details [2]. In this case, the normalized duality mapping $J$ is said to be weakly sequentially continuous, i.e., if $\left\{x_{n}\right\}_{n \in \mathbb{N}} \subset E$ is a sequence with $x_{n} \rightarrow x \in E$, then $J\left(x_{n}\right) \rightarrow^{*} J(x)$ [2]. A Banach space $E$ is said to satisfy the Opial property [3] if for any weakly convergent sequence $\left\{x_{n}\right\}_{n \in \mathbb{N}}$ in $E$ with weak limit $x$,

$$
\limsup _{n \rightarrow \infty}\left\|x_{n}-x\right\|<\limsup _{n \rightarrow \infty}\left\|x_{n}-y\right\|
$$

for all $y \in E$ with $y \neq x$. It is well known that all Hilbert spaces, all finite dimensional Banach spaces, and the Banach spaces $l^{p}(1 \leq p<\infty)$ satisfy the Opial property; see, for example $[2,3]$. It is also known that if $E$ admits a weakly sequentially continuous duality mapping, then $E$ is smooth and enjoys the Opial property; see for more details [2]. Let $C$ a nonempty subset of a real Banach space $E$ and let $T: C \rightarrow E$ be a mapping. The set of fixed points of $T$ is denoted by $F(T)$ and defined by

$$
F(T)=\{x \in C: T x=x\} .
$$

Recall that a mapping $T: C \rightarrow E$ is called nonexpansive if $\|T x-T y\| \leq\|x-y\|$ for all $x, y \in C$. The mapping $T: C \rightarrow E$ is called quasi-nonexpansive, if $F(T) \neq \emptyset$ and

$$
\|p-T x\| \leq\|p-x\|, \quad \forall x \in C, p \in F(T) .
$$

Let $C$ be a nonempty, closed, and convex subset of a Banach space $E$ and $x \in E$. Then there exists a unique nearest point $z \in C$ such that $\|x-z\|=\inf _{y \in C}\|x-y\|$. We denote such a correspondence by $z=P_{C} x$. The mapping $P_{C}$ is called metric projection of $E$ onto $C$.

Let $C$ be a nonempty, closed, and convex subset of a Banach space $E$, let $T$ be a mapping from $C$ into itself. A point $p \in C$ is said to be a weakly asymptotic fixed point [ 4 ] of $T$ if there exists a sequence $\left\{x_{n}\right\}_{n \in \mathbb{N}}$ in $C$ which converges weakly to $p$ and $\lim _{n \rightarrow \infty}\left\|x_{n}-T x_{n}\right\|=0$. We denote the set of all weakly asymptotic fixed points of $T$ by $\hat{F}(T)$. A point $p \in C$ is called a strong asymptotic fixed point of $T$ if there exists a sequence $\left\{x_{n}\right\}_{n \in \mathbb{N}}$ in $C$ which converges strongly to $p$ and $\lim _{n \rightarrow \infty}\left\|x_{n}-T x_{n}\right\|=0$. We denote the set of all strong asymptotic fixed points of $T$ by $\tilde{F}(T)$. Recall that a mapping $T: C \rightarrow C$ is said to be relatively nonexpansive if the following conditions are satisfied: 
(1) $F(T)$ is nonempty;

(2) $\|p-T v\| \leq\|p-v\|, \forall p \in F(T), v \in C$;

(3) $\hat{F}(T)=F(T)$.

A mapping $T: C \rightarrow C$ is said to be weakly relatively nonexpansive if the following conditions are satisfied:

(1) $F(T)$ is nonempty;

(2) $\|p-T v\| \leq\|p-v\|, \forall p \in F(T), v \in C$;

(3) $\tilde{F}(T)=F(T)$.

It is clear that any relatively nonexpansive mapping is a quasi-nonexpansive mapping. It is also obvious that every relatively nonexpansive mapping is a weakly relatively nonexpansive mapping, but the converse in not true in general. Below we show that there exists a weakly relatively nonexpansive mapping which is not a relatively nonexpansive mapping.

Example 1.1 Let $E=l^{2}$, where

$$
\begin{aligned}
& l^{2}=\left\{\sigma=\left(\sigma_{1}, \sigma_{2}, \ldots, \sigma_{n}, \ldots\right): \sum_{n=1}^{\infty}\left\|\sigma_{n}\right\|^{2}<\infty\right\}, \quad\|\sigma\|=\left(\sum_{n=1}^{\infty}\left\|\sigma_{n}\right\|^{2}\right)^{\frac{1}{2}}, \forall \sigma \in l^{2}, \\
& \langle\sigma, \eta\rangle=\sum_{n=1}^{\infty} \sigma_{n} \eta_{n}, \quad \forall \delta=\left(\sigma_{1}, \sigma_{2}, \ldots, \sigma_{n}, \ldots\right), \eta=\left(\eta_{1}, \eta_{2}, \ldots, \eta_{n}, \ldots\right) \in l^{2} .
\end{aligned}
$$

Let $\left\{x_{n}\right\}_{n \in \mathbb{N} \cup\{0\}} \subset E$ be a sequence defined by

$$
\begin{aligned}
& x_{0}=(1,0,0,0, \ldots), \\
& x_{1}=(1,1,0,0,0, \ldots), \\
& x_{2}=(1,0,1,0,0,0, \ldots), \\
& x_{3}=(1,0,0,1,0,0,0, \ldots), \\
& \ldots \\
& x_{n}=\left(\sigma_{n, 1}, \sigma_{n, 2}, \ldots, \sigma_{n, k}, \ldots\right) \\
& \ldots,
\end{aligned}
$$

where

$$
\sigma_{n, k}= \begin{cases}1 & \text { if } k=1, n+1, \\ 0 & \text { if } k \neq 1, k \neq n+1\end{cases}
$$

for all $n \in \mathbb{N}$. Now, we define a mapping $T: E \rightarrow E$ by

$$
T(x)= \begin{cases}\frac{n}{n+1} x, & \text { if } x=x_{n} \\ -x, & \text { if } x \neq x_{n}\end{cases}
$$

Then $F(T)=\{0\}$ and $T$ is a weakly relatively nonexpansive mapping which is not a relatively nonexpansive mapping. Next, we define a countable family of mappings $T_{j}: E \rightarrow E$ 
by

$$
T_{j}(x)= \begin{cases}\frac{n}{n+1} x, & \text { if } x=x_{n} \\ \frac{--1}{j+1} x, & \text { if } x \neq x_{n},\end{cases}
$$

for all $j \geq 1$ and $n \geq 0$. It is clear that $F\left(T_{j}\right)=\{0\}$ for all $j \geq 1$. Choose $j \in \mathbb{N}$, then for any $n \in \mathbb{N}$

$$
\left\|0-T_{j} x_{n}\right\|=\left\|\frac{n}{n+1} x_{n}\right\| \leq\left\|0-x_{n}\right\| .
$$

If $x \neq x_{n}$, then we have

$$
\left\|0-T_{j} x\right\|=\left\|\frac{j}{j+1} x\right\| \leq\|0-x\|
$$

Therefore, $T_{j}$ is a quasi-nonexpansive mapping. By similar arguments to Example 1.1 of [5], we can show that $\hat{F}\left(T_{j}\right) \neq F\left(T_{j}\right)$ for all $j \in \mathbb{N}$. This implies that $\bigcap_{j=1}^{\infty} \hat{F}\left(T_{j}\right) \neq \bigcap_{j=1}^{\infty} F\left(T_{j}\right)$. Thus $\left\{T_{n}\right\}_{n \in \mathbb{N}}$ is a sequence of weakly relatively nonexpansive mappings which is not a sequence of relatively nonexpansive mappings.

Let $E$ be a Banach space and let $E^{*}$ be the dual space of $E$. Let $g: E \rightarrow \mathbb{R}$ be a convex and Gâteaux differentiable function. Then the Bregman distance $[6,7]$ corresponding to $g$ is the function $D_{g}: E \times E \rightarrow \mathbb{R}$ defined by

$$
D_{g}(x, y)=g(x)-g(y)-\langle x-y, \nabla g(y)\rangle, \quad \forall x, y \in E,
$$

where $\nabla g(y)$ stands the value of the gradient $\nabla g$ of $g$ at $y$. It is clear that $D_{g}(x, y) \geq 0$ for all $x, y \in E$. In that case when $E$ is a Hilbert space, setting $g(x)=\frac{1}{2}\|x\|^{2}$ for all $x \in E$, we obtain $\nabla g(x)=2 x$ for all $x \in E$ and hence $D_{g}(x, y)=\frac{1}{2}\|x-y\|^{2}$ for all $x, y \in E$. Let $C$ be a nonempty and convex subset of $E$. Then we know from [8] that for $x \in E$ and $x_{0} \in C$, $D_{g}\left(x_{0}, x\right)=\min _{y \in C} D_{g}(y, x)$ if and only if

$$
\left\langle y-x_{0}, \nabla g(x)-\nabla g\left(x_{0}\right)\right\rangle \leq 0, \quad \forall y \in C .
$$

Furthermore, if $C$ is a nonempty, closed, and convex subset of a reflexive Banach space $E$ and $g: E \rightarrow \mathbb{R}$ is a strongly coercive Bregman function, then for each $x \in E$, there exists a unique $x_{0} \in C$ such that

$$
D_{g}\left(x_{0}, x\right)=\min _{y \in C} D_{g}(y, x)
$$

The Bregman projection $\operatorname{proj}_{C}^{g}$ from $E$ onto $C$ is defined by $\operatorname{proj}_{C}^{g}(x)=x_{0}$ for all $x \in E$. It is also well known that projg has the following property:

$$
D_{g}\left(y, \operatorname{proj}_{C}^{g} x\right)+D_{g}\left(\operatorname{proj}_{C}^{g} x, x\right) \leq D_{g}(y, x)
$$

for all $y \in C$ and $x \in E$ (see [9] for more details). 
Remark 1.1 It is worth mentioning that the class of weakly relatively nonexpansive mappings introduced in the present paper is different from the class of Bregman weak relatively nonexpansive mappings introduced in [5]. It is well known that in a Banach space $E$ the Bregman projection operator $\operatorname{proj}_{C}^{g}$ is a Bregman weak relatively nonexpansisive mapping but it is not a quasi-nonexpansive mapping with respect to the norm of the space; see, for example, $[5,9]$.

Moudafi [10] introduced the following iterative process, which is called Moudafi's viscosity approximation: $x_{1}=x \in C$ and

$$
x_{n+1}=\alpha_{n} f\left(x_{n}\right)+\left(1-\alpha_{n}\right) T x_{n}
$$

for all $n \in \mathbb{N}$, where $\left\{\alpha_{n}\right\}_{n \in \mathbb{N}} \subset[0,1)$ and $f: C \rightarrow C$ is a contraction. See also [11]. It was proved that this sequence converges strongly to a unique fixed point of $P_{F} f$ under similar conditions to those in [12]. Suzuki [13] considered the Meir-Keeler contractions, which is an extended notion of contractions, and studied the equivalency of convergence of these approximation schemes.

Let $C$ be a nonempty, closed, and convex subset of a reflexive Banach space $E$. Let $n \in \mathbb{N}$ and $T_{n}: C \rightarrow C$ be a quasi-nonexpansive mapping. For given $x \in C$, let $\left\{x_{n}\right\}_{n \in \mathbb{N}}$ be generated by the algorithm

$$
x_{n+1}:=\alpha_{n} f\left(x_{n}\right)+\left(1-\alpha_{n}\right) T_{n}\left(x_{n}\right), \quad n \geq 0,
$$

where $\left\{\alpha_{n}\right\}_{n \in \mathbb{N}} \subset(0,1)$ satisfies the following conditions: (i) $\lim _{n \rightarrow \infty} \alpha_{n}=0$; (ii) $\sum_{n=1}^{\infty} \alpha_{n}=$ $\infty$. Then $\left\{x_{n}\right\}_{n \in \mathbb{N}}$ is said to satisfy condition (A) if for any subsequence $x_{n_{k}} \rightarrow x$ and $x_{n+1}-T_{n}\left(x_{n}\right) \rightarrow 0$ implies that $x \in F:=\bigcap_{n=1}^{\infty} F\left(T_{n}\right)$; for more details, see [14]. Motivated by the above notation, we say that the sequence $\left\{x_{n}\right\}_{n \in \mathbb{N}}$ satisfies condition (B) if for any subsequence $x_{n_{k}} \rightarrow x$ and $x_{n+1}-T_{n}\left(x_{n}\right) \rightarrow 0$ implies that $x \in F:=\bigcap_{n=1}^{\infty} F\left(T_{n}\right)$. It is clear that if the sequence $\left\{x_{n}\right\}_{n \in \mathbb{N}}$ satisfies condition (B), then it satisfies condition (A), however, the converse is not true in general. For instance, the sequence $\left\{x_{n}\right\}_{n \in \mathbb{N}}$ defined in Example 1.1 satisfies condition (B) but it does not satisfy condition (A). Indeed, $\lim _{n \rightarrow \infty}\left\|x_{n+1}-T_{n} x_{n}\right\|=\lim _{n \rightarrow \infty}\left\|\frac{n+1}{n+2} x-\frac{n}{n+1} x\right\|=\lim _{n \rightarrow \infty}\left\|\frac{1}{(n+1)(n+2)} x\right\|=0$ and the sequence $\left\{x_{n}\right\}_{n \in \mathbb{N}}$ converges weakly to $x_{0}$ but $x_{0} \notin \bigcap_{n=1}^{\infty} F\left(T_{n}\right)$.

Definition 1.1 Let $E$ be a real Banach space and $D$ be a closed subset of $E$. A mapping $T: D \rightarrow D$ is said to be demiclosed at the origin if, for any sequence $\left\{x_{n}\right\}_{n \in \mathbb{N}}$ in $D$, the conditions $x_{n} \rightarrow x_{0}$ and $T x_{n} \rightarrow 0$ imply $T x_{0}=0$.

Let $C$ and $D$ be nonempty subsets of real Banach space $E$ with $D \subset C$. A mapping $Q_{D}$ : $C \rightarrow D$ is said to be sunny if

$$
Q_{D}\left(Q_{D} x+t\left(x-Q_{D} x\right)\right)=Q_{D} x
$$

for each $x \in E$ and $t \geq 0$. A mapping $Q_{D}: C \rightarrow D$ is said to be a retraction if $Q_{D} x=x$ for each $x \in C$.

Recently, Zegeye and Shahzad [14] proved the following fixed point theorem for quasinonexpansive mappings in a Banach space. 
Theorem 1.1 Let $K$ be a nonempty, closed, and convex subset of a real reflexive Banach space $E$ that has weakly continuous duality mapping $J_{\phi}$ for some gauge $\phi$. Let $T_{i}: K \rightarrow K$, $i=1,2, \ldots$, be a family of quasi-nonexpansive mappings with $F:=\bigcap_{i=1}^{\infty} F\left(T_{i}\right) \neq \emptyset$; which is a sunny nonexpansive retract of $K$ with $Q$ a nonexpansive retraction. For given $x_{0} \in K$, let $\left\{x_{n}\right\}_{n \in \mathbb{N}}$ be generated by the algorithm

$$
x_{n+1}:=\alpha_{n} f\left(x_{n}\right)+\left(1-\alpha_{n}\right) T_{n}\left(x_{n}\right), \quad n \geq 0,
$$

where $f: K \rightarrow K$ is a contraction mapping with constant $\beta \in(0,1)$ and $\left\{\alpha_{n}\right\}_{n \in \mathbb{N}} \subset(0,1)$ satisfies the following conditions:

(i) $\lim _{n \rightarrow \infty} \alpha_{n}=0$;

(ii) $\sum_{n=1}^{\infty} \alpha_{n}=\infty$.

Suppose that $\left\{x_{n}\right\}_{n \in \mathbb{N}}$ satisfies condition (A). Then $\left\{x_{n}\right\}_{n \in \mathbb{N}}$ converges strongly to a common fixed point $\bar{x}=Q(f(\bar{x}))$ of a family $T_{i}, i=1,2, \ldots$, as $n \rightarrow \infty$. Moreover, $\bar{x}$ is the unique solution in $F$ to the variational inequality

$$
\left\langle f(\bar{x})-\bar{x}, J_{\phi}(y-\bar{x})\right\rangle \leq 0, \quad \forall y \in F .
$$

Very recently, Maingé [15] studied strong convergence theorems for quasi-nonexpansive mappings and posed the following open problem in his final remark.

Question 1.1 Is there any strong convergence theorem of Moudafi's type for quasinonexpansive mappings without using the demiclosedness principle in a Banach space $E$ ?

Many problems in nonlinear analysis can be formulated as a problem of finding a fixed point of a nonexpansive-type mapping. There exists an extensive literature regarding the convergence analysis of iterative methods for approximation fixed points of several types of mappings $T$, in the settings of Hilbert and Banach spaces (see, e.g., [16-21]). However, to the best of our knowledge, there is no strong convergence result regarding the viscosity approximation of a weakly relatively nonexpansive mapping in a Banach space. In this paper, we first introduce a new viscosity approximation method based on the shrinking projection algorithm to approximate a common fixed point of a countable family of nonlinear mappings in a Banach space. Under quite mild assumptions, we establish the strong convergence of the sequence generated by the proposed algorithm. In contrast with other related processes, our method does not require any demiclosedness principle condition imposed on the involved operators belonging to the vide class of quasi-nonexpansive operators. As an application, we also introduce an iterative algorithm for finding a common element of the set of common fixed points of an infinite family of quasi-nonexpansive mappings and the set of solutions of a mixed equilibrium problem in a real Banach space. We prove a strong convergence theorem by using the proposed algorithm under some suitable conditions. Our results improve and generalize many known results in the current literature; see, for example, [14, 15, 22].

\section{Preliminaries}

In this section, we collect some lemmas which will be used in the proofs for the main results in next sections. 
Lemma 2.1 [21] Let $C$ and $D$ be nonempty subsets of a real Banach space $E$ with $D \subset C$ and let $Q_{D}: C \rightarrow D$ be a retraction from $C$ into $D$. Then $Q_{D}$ is sunny and nonexpansive if and only if

$$
\left\langle z-Q_{D}(z), J\left(y-Q_{D}(z)\right)\right\rangle \leq 0
$$

for all $z \in C$ and $y \in D$, where $J$ is the normalized duality mapping of $E$.

Lemma 2.2 [21] Let E be a real Banach space and $J$ be the normalized duality mapping of E. Then

$$
\|x+y\|^{2} \leq\|x\|^{2}+2\langle y, J(x+y)\rangle
$$

for all $x, y \in E$.

Theorem 2.1 A Meir-Keeler contraction defined on a complete metric space has a unique fixed point. We have the following result, given by Suzuki [13], for Meir-Keeler contractions defined on a Banach space.

Lemma 2.3 (Suzuki [13]) Let $C$ be a nonempty convex subset of a Banach space E and $f: C \rightarrow E$ be a Meir-Keeler contraction. Then, for every $\epsilon>0$, there exists $r \in(0,1)$ such that $\|x-y\| \geq \epsilon$ implies that $\|f(x)-f(y)\| \leq r\|x-y\|$ for $x, y \in C$.

Let $\left\{C_{n}\right\}_{n \in \mathbb{N}}$ be a sequence of nonempty, closed, and convex subsets of a reflexive Banach space $E$. We define a subset s- $\mathrm{Li}_{n} C_{n}$ of $E$ as follows: $x \in \mathrm{s}-\mathrm{Li}_{n} C_{n}$ if and only if there exists $\left\{x_{n}\right\}_{n \in \mathbb{N}} \subset E$ such that $\left\{x_{n}\right\}_{n \in \mathbb{N}}$ converges strongly to $x$ and such that $x_{n} \in C_{n}$ for all $n \in \mathbb{N}$. Similarly, a subset $\mathrm{w}-\mathrm{Ls}_{n} C_{n}$ of $E$ is defined by the following: $y \in \mathrm{w}-\mathrm{Ls}_{n} C_{n}$ if and only if there exist a subsequence $\left\{C_{n_{i}}\right\}_{i \in \mathbb{N}}$ of $\left\{C_{n}\right\}_{n \in \mathbb{N}}$ and a sequence $\left\{y_{i}\right\}_{i \in \mathbb{N}} \subset E$ such that $\left\{y_{i}\right\}_{i \in \mathbb{N}}$ converges weakly to $y$ and such that $y_{i} \in C_{n_{i}}$ for all $i \in \mathbb{N}$. If $C_{0} \subset E$ satisfies $C_{0}=\mathrm{s}-\mathrm{Li}_{n} C_{n}=\mathrm{w}-\mathrm{Ls}_{n} C_{n}$, it is said that $\left\{C_{n}\right\}_{n \in \mathbb{N}}$ converges to $C_{0}$ in the sense of Mosco [23], and we write $C_{0}=\mathrm{M}-\lim _{n} C_{n}$. One of the simplest examples of Mosco convergence is a decreasing sequence $\left\{C_{n}\right\}_{n \in \mathbb{N}}$ with respect to inclusion. The Mosco limit of such a sequence is $\bigcap_{n=1}^{\infty} C_{n}$. For more details, see [24]. Tsukada [25] proved the following theorem for the metric projection in a Banach space.

Theorem 2.2 (Tsukada [25]) Let $\left\{C_{n}\right\}_{n \in \mathbb{N}}$ be a sequence of nonempty, closed, and convex subsets of a Banach space E. If $C_{0}=\mathrm{M}-\lim _{n} C_{n}$ exists and is nonempty, then, for each $x \in E$, $\left\{P_{C_{n}} x\right\}_{n \in \mathbb{N}}$ converges strongly to $P_{C_{0}} x$.

\section{Strong convergence theorems}

In this section, we prove a strong convergence theorem for approximating common fixed points of weakly relatively nonexpansive mappings in a Banach space.

Theorem 3.1 Let $C$ be a nonempty, closed and convex subset of a Banach space $E$ and $\left\{T_{n}\right\}_{n \in \mathbb{N}}$ an infinite family of weakly relatively nonexpansive mappings from $C$ into itself with $F:=\bigcap_{n=1}^{\infty} F\left(T_{n}\right) \neq \emptyset$ which is a sunny nonexpansive retract of $C$ with $Q$ a nonexpansive 
retraction. Let $\left\{x_{n}\right\}_{n \in \mathbb{N} \cup\{0\}}$ be a sequence generated by

$$
\left\{\begin{array}{l}
x_{0}=x \in C \quad \text { chosen arbitrarily, } \\
C_{0}=D_{0}=C, \\
C_{n+1}=\left\{z \in C_{n}:\left\|z-T_{n} x_{n}\right\| \leq\left\|z-x_{n}\right\|\right\}, \\
D_{n+1}=\overline{c o} C_{n+1}, \\
P_{D_{n+1} x_{n}=T_{n} x_{n},} \\
x_{n+1}=Q_{D_{n+1}} f\left(x_{n}\right) \text { and } n \in \mathbb{N} \cup\{0\} .
\end{array}\right.
$$

Then there exists $v \in C$ such that $\left\{x_{n}\right\}_{n \in \mathbb{N}}$ and $\left\{T_{n} x_{n}\right\}_{n \in \mathbb{N}}$, converge strongly to $Q_{F} f(v)=v$.

Proof In the light of [13, Proposition 3], we see that the composed mapping $Q_{F} f$ of $C$ into itself is a Meir-Keeler contraction on $C$. Theorem 2.1 implies that there exists a unique fixed point $v \in F$ of $Q_{F} f$. Since $D_{n}$ is a closed and convex subset of $E$ and $\emptyset \neq D_{n+1} \subset D_{n}$ for all $n \in \mathbb{N},\left\{x_{n}\right\}_{n \in \mathbb{N}}$ is well defined. On the other hand, the composed mapping $Q_{\bigcap_{n=1}^{\infty} D_{n}} f$ is a Meir-Keeler contraction on $C$, so in light of Theorem 2.1 there exists a unique fixed point $v \in \bigcap_{n=1}^{\infty} D_{n}$ of $Q_{\bigcap_{n=1}^{\infty} D_{n}} f$. Furthermore, setting $z_{n}=Q_{D_{n}} f(v)$ for each $n \in \mathbb{N}$ and noting that $F \subset D_{n+1} \subset D_{n}$ for every $n \in \mathbb{N}$, we immediately obtain $\bigcap_{n=1}^{\infty} D_{n}=\mathrm{M}-\lim _{n} D_{n}$. Thus, by Theorem 2.2,

$$
z_{n} \rightarrow Q_{\bigcap_{n=1}^{\infty} D_{n}} f(v)=v
$$

Next, we prove that $x_{n} \rightarrow v$ as $n \rightarrow \infty$. Suppose on the contrary that $\lim _{\sup _{n \rightarrow \infty}}\left\|x_{n}-u\right\|>$ 0 . Then there exists $\epsilon>0$ such that $\lim _{\sup _{n \rightarrow \infty}}\left\|x_{n}-v\right\|>0$. By the definition of MeirKeeler contraction, there exists $\delta>0$ with $\delta+\epsilon<\lim \sup _{n \rightarrow \infty}\left\|x_{n}-v\right\|$ such that $\|x-y\|<$ $\delta+\epsilon$ implies that $\|f(x)-f(y)\|<\epsilon$ for all $x, y \in C$. From Lemma 2.3, there exists $r \in(0,1)$ such that $\|x-y\| \geq \delta+\epsilon$ implies that $\|f(x)-f(y)\| \leq r\|x-y\|$ for every $x, y \in C$. Then, from (3.2), we can choose $n_{0} \in \mathbb{N}$ such that $\left\|z_{n}-v\right\|<\delta$ for each $n \geq n_{0}$. As in the proof of [13, Theorem 8], we consider the following two cases.

(i) There exists $n_{1} \geq n_{0}$ such that $\left\|x_{n_{1}}-v\right\|<\delta+\epsilon$.

(ii) $\|x-y\| \geq \delta+\epsilon$ for every $n \geq n_{0}$.

In case (i), we have $\left\|x_{n_{1}+1}-z_{n_{1}+1}\right\| \leq\left\|f\left(x_{n_{1}}\right)-f(v)\right\|<\epsilon$ since $\left\|x_{n_{1}}-y\right\| \geq \delta+\epsilon$. It follows immediately that $\left\|x_{n_{1}+1}-v\right\| \leq\left\|x_{n_{1}+1}-z_{n_{1}+1}\right\|+\left\|z_{n_{1}+1}-v\right\|<\delta+\epsilon$. This means that

$$
\limsup _{n \rightarrow \infty}\left\|x_{n}-v\right\| \leq \delta+\epsilon<\limsup _{n \rightarrow \infty}\left\|x_{n}-v\right\| .
$$

This is a contradiction. In case (ii), we have $\left\|f\left(x_{n}\right)-f(u)\right\| \leq r\left\|x_{n}-u\right\|$ for all $n \geq n_{0}$. Thus we get

$$
\left\|x_{n+1}-z_{n+1}\right\| \leq\left\|f\left(x_{n}\right)-f(v)\right\| \leq r\left\|x_{n}-v\right\| \leq r\left(\left\|x_{n}-z_{n}\right\|+\left\|z_{n}-v\right\|\right)
$$

for every $n \geq n_{0}$. This latter result together with (3.2) amounts to

$$
\limsup _{n \rightarrow \infty}\left\|x_{n}-z_{n}\right\|=\limsup _{n \rightarrow \infty}\left\|x_{n+1}-z_{n+1}\right\| \leq r \limsup _{n \rightarrow \infty}\left\|x_{n}-z_{n}\right\|<\limsup _{n \rightarrow \infty}\left\|x_{n}-z_{n}\right\| .
$$

This is a contradiction. Therefore, we get

$$
\lim _{n \rightarrow \infty} x_{n}=v
$$


Since $x_{n+1}=Q_{D_{n+1}} f\left(x_{n}\right)$, we have $\left\langle f\left(x_{n}\right)-x_{n+1}, x_{n+1}-y\right\rangle \geq 0$ for each $y \in D_{n+1}$. Using $F \subset$ $D_{n+1}$, we get $\left\langle f\left(x_{n}\right)-x_{n+1}, x_{n+1}-y\right\rangle \geq 0$ for every $n \in \mathbb{N}$ and $y \in F$, which implies that

$$
\langle f(v)-v, v-y\rangle \geq 0
$$

for each $y \in F$. On the other hand, since $P_{D_{n+1}} x_{n}=T_{n} x_{n}$ and $x_{n+1} \in D_{n+1}$, we have

$$
\left\|x_{n}-T_{n} x_{n}\right\| \leq\left\|x_{n}-x_{n+1}\right\|
$$

It turns out that

$$
\lim _{n \rightarrow \infty}\left\|x_{n}-T_{n} x_{n}\right\|=\lim _{n \rightarrow \infty}\left\|x_{n}-x_{n+1}\right\|=0 .
$$

It follows that $v \in \bigcap_{n=1}^{\infty} F\left(T_{n}\right)$. Hence $v$ is a strong limit of $\left\{x_{n}\right\}_{n \in \mathbb{N}}$ and $\left\{T_{n} x_{n}\right\}_{n \in \mathbb{N}}$, which completes the proof.

Remark 3.1 (1) Theorem 3.1 extends and improves Theorem 1.1. We did not use the demiclosedness principle (the condition (A) of the sequence $\left\{x_{n}\right\}_{n \in \mathbb{N}}$ ) in our discussion. Our Theorem 3.1 is also valid in a wide class of general Banach spaces while Theorem 1.1 is valid in Banach spaces having weakly sequentially duality mappings.

(2) We note also that the main result of the paper provides a positive answer to open Question 1.1. So, our Theorem 3.1 improves the main result of [15] from a Hilbert space to a Banach space.

\section{Application to equilibrium problems}

The equilibrium problem was first introduced by Fan in [26] (see, also [27]). It is well known that the equilibrium problem includes many important problems in nonlinear analysis and optimization such as the Nash equilibrium problem, variational inequalities, complementarity problems, vector optimization problems, fixed point problems, saddle point problems, and game theory; see for example [28] and its references. Existence results for solutions to equilibrium problems have been extensively studied, as can be seen in $[27,28]$.

Let $C$ be a nonempty, closed, and convex subset of a Banach space $E$. Let $h: C \times C \rightarrow$ $\mathbb{R}$ be a bifunction and let $\phi: C \rightarrow \mathbb{R}$ be a function. Consider the following equilibrium problem: Find $p \in C$ such that

$$
h(p, y) \geq 0, \quad \forall y \in C .
$$

The mixed equilibrium problem is to find $q \in C$ such that

$$
h(q, y)+\phi(y) \geq \phi(x), \quad \forall y \in C .
$$

Problem (4.2) was first introduced by Ceng and Yao [29]. The solution set of (4.2) is denoted by $M E P(h, \phi)$. If $\phi \equiv 0$, then the mixed equilibrium problem (4.2) reduces to the equilibrium problem (4.1).

For solving the mixed equilibrium problem, let us assume that $h: C \times C \rightarrow \mathbb{R}$ satisfies the following conditions: 
(A1) $h(x, x)=0$ for all $x \in C$;

(A2) $h$ is monotone, i.e., $h(x, y)+h(y, x) \leq 0$ for all $x, y \in C$;

(A3) for all $y \in C, h(\cdot, y)$ is weakly upper semicontinuous;

(A4) for all $x \in C, h(x, \cdot)$ is convex.

In this section, we prove a strong convergence theorem concerning equilibrium problems in a Banach space. Let $E$ be a smooth, strictly convex, and reflexive Banach space. Let $C$ be a nonempty, bounded, closed, and convex subset of $E$, let $h$ be a bifunction from $C \times C$ to $\mathbb{R}$ satisfying (A1)-(A4) and let $\phi$ be a lower semicontinuous and convex function from $C$ to $\mathbb{R}$. For all $r>0$, we define the mapping $T_{r}: E \rightarrow 2^{C}$ as follows:

$$
T_{r}(x)=\left\{z \in C: h(z, y)+\phi(y)+\frac{1}{r}\langle y-z, J(z-x)| \geq \phi(z) \text { for all } y \in C\right\}
$$

for all $x \in E$.

Lemma 4.1 [30] Let E be a Banach space and $C$ be a nonempty, bounded, closed, and convex subset of $E$ and $h: C \times C \rightarrow \mathbb{R}$ a bifunction satisfying (A1)-(A4) and $M E P(h, \phi) \neq \emptyset$. For $r>0$, let $T_{r}: E \rightarrow C$ be the mapping defined by (4.3). Then the following statements hold:

(1) $T_{r}(x)$ is nonempty for every $x \in E$;

(2) $T_{r}$ is single-valued;

(3) $\left\langle T_{r} x-T_{r} y, J\left(T_{r} x-x\right)\right\rangle \leq\left\langle T_{r} x-T_{r} y, J\left(T_{r} y-y\right)\right\rangle$, for all $x, y \in E$;

(4) $F\left(T_{r}\right)=M E P(h, \phi)$;

(5) $\operatorname{MEP}(h, \phi)$ is nonempty, closed, and convex.

Theorem 4.1 Let $E$ be a uniformly convex and smooth Banach space and let $C$ be a nonempty, bounded, closed, and convex subset of $E$. Let h be a bifunction from $C \times C$ to $\mathbb{R}$ satisfying (A1)-(A4), let $\phi$ be a lower semicontinuous and convex function from $C$ to $\mathbb{R}$ and let $\left\{T_{n}\right\}_{n \in \mathbb{N}}$ be a sequence of weakly relatively nonexpansive mappings of $C$ into itself such that $F:=\bigcap_{n=1}^{\infty} F\left(T_{n}\right) \cap M E P(h, \phi) \neq \emptyset$ which is a sunny nonexpansive retract of $C$ with $Q$ a nonexpansive retraction. For $r>0$, let $T_{r}: E \rightarrow C$ be the mapping defined by (4.3). Let $\left\{r_{n}\right\}_{n \in \mathbb{N}}$ be a sequence in $(0, \infty)$ such that $\liminf _{n \rightarrow \infty} r_{n}>0$. Let $\left\{x_{n}\right\}_{n \in \mathbb{N}}$ be a sequence generated by

$$
\left\{\begin{array}{l}
x_{0}=x \in C \text { chosen arbitrarily, } \\
C_{0}=D_{0}=C \\
C_{n+1}=\left\{z \in C_{n}:\left\|z-T_{n} x_{n}\right\| \leq\left\|z-x_{n}\right\|\right\}, \quad n \geq 0, \\
D_{n+1}=\overline{c o} C_{n+1}, \\
P_{D_{n+1}} x_{n}=T_{n} x_{n}, \\
Q_{n+1}=\left\{z \in Q_{n}:\left\langle T_{r_{n}} x_{n}-z, J\left(x_{n}-T_{r_{n}} x_{n}\right)\right\rangle \geq 0\right\}, \quad n \geq 1, \\
x_{n+1}=Q_{D_{n+1} \cap Q_{n+1}} f\left(x_{n}\right) \text { and } n \in \mathbb{N} \cup\{0\} .
\end{array}\right.
$$

Then there exists $v \in C$ such that $\left\{x_{n}\right\}_{n \in \mathbb{N}}$ converges strongly to $Q_{F} f(v)=v$.

Proof We first show that the sequence $\left\{x_{n}\right\}_{n \in \mathbb{N}}$ is well defined. It is easy to verify that $D_{n} \cap$ $Q_{n}$ is closed and convex and $F \subset D_{n}$ for all $n \geq 0$. Since $D_{0}=C$, we also have $F \subset D_{0} \cap Q_{0}$. 
Suppose that $F \subset D_{m-1} \cap Q_{m-1}$ for $m \geq 2$. It follows from Lemma 4.1(3) that

$$
\left\langle T_{r_{m}} x_{m}-T_{r_{m}} u, J\left(T_{r_{m}} u-u\right)-J\left(T_{r_{m}} x_{m}-x_{m}\right)\right\rangle \geq 0 \quad \text { for all } u \in F
$$

This implies that

$$
\left\langle T_{r_{m}} x_{m}-u, J\left(x_{m}-T_{r_{m}} x_{m}\right)\right\rangle \geq 0,
$$

for all $u \in F$. Hence $F \subset Q_{m}$. By mathematical induction, we get $F \subset D_{n} \cap Q_{n}$ for each $n \geq 0$ and hence $\left\{x_{n}\right\}_{n \in \mathbb{N}}$ is well defined.

Since $Q_{F}$ is nonexpansive, the composed mapping $Q_{F} f$ of $C$ into itself is a Meir-Keeler contraction on $C$; see [13, Proposition 3]. In view of Theorem 2.1, there exists a unique fixed point $v \in F$ of $Q_{F} f$. Since $D_{n}$ is a closed and convex subset of $E$ and $\emptyset \neq D_{n+1} \subset D_{n}$ for all $n \in \mathbb{N},\left\{x_{n}\right\}_{n \in \mathbb{N}}$ is well defined. On the other hand, the composed mapping $Q_{\bigcap_{n=1}^{\infty} D_{n}} f$ is a Meir-Keeler contraction on $C$, so in view of Theorem 2.1 there exists a unique fixed point $v \in \bigcap_{n=1}^{\infty} D_{n}$ of $Q_{\bigcap_{n=1}^{\infty} D_{n}} f$. Furthermore, setting $z_{n}=Q_{D_{n}} f(v)$ for each $n \in \mathbb{N}$ and noting that $F \subset D_{n+1} \subset D_{n}$ for every $n \in \mathbb{N}$, we immediately obtain $\bigcap_{n=1}^{\infty} D_{n}=\mathrm{M}-\lim _{n} D_{n}$. Thus, by Theorem 2.2,

$$
z_{n} \rightarrow Q_{\bigcap_{n=1}^{\infty} D_{n}} f(v)=v
$$

By the same arguments, as in the proof of Theorem 3.1, we can prove that $x_{n} \rightarrow v$ as $n \rightarrow \infty$.

Next, we show that $v \in M E P(h, \phi)$. By the construction of $Q_{n}$, we see from (4.4) that $T_{r_{n}} x_{n}=P_{Q_{n}} x_{n}$. Since $x_{n+1} \in Q_{n}$, we obtain

$$
\left\|x_{n}-T_{r_{n}} x_{n}\right\| \leq\left\|x_{n}-x_{n+1}\right\|
$$

which together with (4.5) leads to

$$
\lim _{n \rightarrow \infty}\left\|x_{n}-T_{r_{n}} x_{n}\right\|=0
$$

From the assumption $\liminf _{n \rightarrow \infty} r_{n}>0$, we also have

$$
\lim _{n \rightarrow \infty} \frac{1}{r_{n}}\left\|J\left(x_{n}-r_{n} x_{n}\right)\right\|=\lim _{n \rightarrow \infty} \frac{1}{r_{n}}\left\|\left(x_{n}-r_{n} x_{n}\right)\right\|=0 .
$$

Since $x_{n} \rightarrow v$, we also have $T_{r_{n}} x_{n} \rightarrow v$ as $n \rightarrow \infty$. By the definition of $T_{r_{n}}$, for each $y \in C$, we deduce that

$$
h\left(T_{r_{n}} x_{n}, y\right)+\phi(y)+\frac{1}{r_{n}}\left\langle y-T_{r_{n}} x_{n}, J\left(T_{r_{n}} x_{n}-x_{n}\right)\right\rangle \geq \phi\left(T_{r_{n}} x_{n}\right) .
$$

By (A3), (4.6), and the weakly lower semicontinuity of $\phi$, we have

$$
h(v, y)+\phi(y) \geq \phi(v), \quad \forall y \in C .
$$

This shows that $v \in \operatorname{MEP}(h, \phi)$ and hence $v \in F=\bigcap_{n=1}^{\infty} F\left(T_{n}\right) \cap M E P(h, \phi)$. 
Remark 4.1 The main result of [30] gave a strong convergence theorem to approximate fixed point of an infinite family of nonexpansive mappings, while the main result of the present paper gives a strong convergence theorem to approximate common fixed points of an infinite family of weakly relatively nonexpansive mappings in a uniformly convex Banach space. We note that the proof of Theorem 3.1 (lines 24-25, where the authors used the nonexpansivity of the mapping $T$ ) in [30] is not valid in our discussion. So our result extends and improves the corresponding results of [30].

Remark 4.2 (1) In Theorem 4.1, we present a strong convergence result for a system of equilibrium problems with new algorithms and new control conditions. This is complementary to the main results of [31-34]. In addition, our scheme in Theorem 4.1 has an advantage that it does not require any demiclosedness principle condition imposed on the involved operators belonging to the wide class of quasi-nonexpansive operators. Indeed, we propose different approaches, based on shrinking projection algorithms, to solve the equilibrium problem in a Banach space. So, our Theorem 4.1 improves the main results of [31-34].

\section{Competing interests}

The authors declare that they have no competing interests.

\section{Authors' contributions}

All authors contributed equally to the writing of this paper. All authors read and approved the final manuscript.

\section{Author details}

'Department of Information Management, Yuan Ze University, Chung-Li, 32003, Taiwan. ${ }^{2}$ Department of Mathematics, Yasouj University, Yasouj, 75918, Iran. ${ }^{3}$ Center for Fundamental Science, Kaohsiung Medical University, Kaohsiung, 807, Taiwan.

\section{Acknowledgements}

This research was partially supported by a grant from NSC.

Received: 29 August 2014 Accepted: 27 December 2014 Published online: 03 February 2015

\section{References}

1. Takahashi, W: Nonlinear Functional Analysis, Fixed Point Theory and Its Applications. Yokohama Publishers, Yokohama (2000)

2. Gossez, JP, Dozo, EL: Some geometric properties related to the fixed point theory for nonexpansive mappings. Pac. J. Math. 40, 565-573 (1972)

3. Geobel, K, Kirk, WA: In: Topics on Metric Fixed-Point Theory. Cambridge Studies in Advanced Mathematics, vol. 28 Cambridge University Press, Cambridge (1990)

4. Reich, S: A weak convergence theorem for the altering method with Bregman distances. In: Theory and Applications of Nonlinear Operators of Accretive and Monotone Type, pp. 313-318. Dekker, New York (1996)

5. Naraghirad, E, Yao, J-C: Bregman weak relatively nonexpansive mappings in Banach spaces. Fixed Point Theory Appl. 2013, 141 (2013)

6. Bregman, LM: The relation method of finding the common point of convex sets and its application to the solution of problems in convex programming. USSR Comput. Math. Math. Phys. 7, 200-217 (1967)

7. Censor, Y, Lent, A: An iterative row-action method for interval convex programming. J. Optim. Theory Appl. 34, 321-358 (1981)

8. Naraghirad, E, Takahashi, W, Yao, J-C: Generalized retraction and fixed point theorems using Bregman functions in Banach spaces. J. Nonlinear Convex Anal. 13(1), 141-156 (2012)

9. Butnariu, D, lusem, AN: Totally Convex Functions for Fixed Points Computation and Infinite Dimensional Optimization. Kluwer Academic, Dordrecht (2000)

10. Moudafi, A: Viscosity approximation methods for fixed-points problems. J. Math. Anal. Appl. 241, $46-55$ (2000)

11. Xu, HK: Viscosity approximation methods for nonexpansive mappings. J. Math. Anal. Appl. 298, $279-291$ (2004)

12. Wittmann, R: Approximation of fixed points of nonexpansive mappings. Arch. Math. (Basel) 58, $486-491$ (1992)

13. Suzuki, T: Moudafi viscosity approximations with Meir-Keeler contractions. J. Math. Anal. Appl. 325, 342-352 (2007)

14. Zegeye, $\mathrm{H}$, Shahzad, $\mathrm{N}$ : Viscosity methods of approximation for a common fixed point of a family of quasi-nonexpansive mappings. Nonlinear Anal. 68, 2005-2012 (2008)

15. Maingé, P-E: The viscosity approximation process for quasi-nonexpansive mappings in Hilbert spaces. Comput. Math. Appl. 59, 74-79 (2010)

16. Mann, WR: Mean value methods in iteration. Proc. Am. Math. Soc. 4, 506-510 (1953) 
17. Halpern, B: Fixed points of nonexpanding mappings. Bull. Am. Math. Soc. 73, 957-961 (1967)

18. Reich, S: Weak convergence theorems for nonexpansive mappings in Banach spaces. J. Math. Anal. Appl. 67, 274-276 (1979)

19. Aoyama, K, Kohsaka, F, Takahashi, W: Shrinking projection methods for firmly nonexpansive mappings. Nonlinear Anal. 71, e1626-e1632 (2009)

20. Genel, A, Lindenstrauss, J: An example concerning fixed points. Isr. J. Math. 22, 81-86 (1975)

21. Goebel, K, Reich, S: Uniform Convexity, Hyperbolic Geometry, and Nonexpansive Mappings. Dekker, New York (1984)

22. Kimura, Y, Nakajo, K: Viscosity approximations by the shrinking projection method in Hilbert spaces. Comput. Math. Appl. 63, 1400-1408 (2012)

23. Mosco, U: Convergence of convex sets and of solutions of variational inequalities. Adv. Math. 3, 510-585 (1969)

24. Beer, G: Topologies on Closed and Closed Convex Sets. Kluwer Academic, Dordrecht (1993)

25. Tsukada, M: Convergence of best approximations in a smooth Banach space. J. Approx. Theory 40, 301-309 (1984)

26. Fan, K: A minimax inequality and applications. In: Shisha, O (ed.) Inequalities III. Academic Press, New York (1972)

27. Blum, E, Oettli, W: From optimization and variational inequalities to equilibrium problems. Math. Stud. 62, 127-169 (1994)

28. Iusem, AN, Kassay, G, Sosa, W: On certain conditions for the existence of solutions of equilibrium problems. Math. Program. 116, 259-273 (2009)

29. Ceng, L-C, Yao, J-C: A hybrid iterative scheme for mixed equilibrium problems and fixed point problems. J. Comput. Appl. Math. 214, 186-201 (2008)

30. Cholamjiak, P, Suantai, S: Existence and iteration for a mixed equilibrium problem and countable family of nonexpansive mappings in Banach spaces. Comput. Math. Appl. 61, 2725-2733 (2011)

31. Ceng, L-C, Ansari, QH, Yao, J-C:Viscosity approximation methods for generalized equilibrium problems and fixed point problems. J. Glob. Optim. 43(4), 487-502 (2009)

32. Ceng, L-C, Ansari, QH, Yao, J-C: Hybrid proximal-type and hybrid shrinking projection algorithms for equilibrium problems, maximal monotone operators and relatively nonexpansive mappings. Numer. Funct. Anal. Optim. 31(7), 763-797 (2010)

33. Ceng, L-C, Ansari, QH, Schaible, S, Yao, J-C: Hybrid viscosity approximate method for zeros of $m$-accretive operators in Banach spaces. Numer. Funct. Anal. Optim. 33(2), 142-165 (2012)

34. Ceng, L-C, Al-Otaibi, A, Ansari, QH, Latif, A: Relaxed and composite viscosity methods for variational inequalities, fixed points of nonexpansive mappings and zeros of accretive operators. Fixed Point Theory Appl. 2014, 29 (2014)

\section{Submit your manuscript to a SpringerOpen ${ }^{\circ}$ journal and benefit from:}

- Convenient online submission

Rigorous peer review

- Immediate publication on acceptance

- Open access: articles freely available online

- High visibility within the field

- Retaining the copyright to your article 